\title{
An Estimate of Hospitalization Cost for Elderly Patients with Acute Upper Gastrointestinal Bleeding in the USA
}

\author{
Amer A. Alkhatib · Samer M. Abubakr • \\ Fateh A. Elkhatib
}

Published online: 3 December 2008

(C) Springer Science+Business Media, LLC 2008

Dear Editor,

The cost of acute upper gastrointestinal bleeding (AUGIB) in the United States was estimated to be around $\$ 2.5$ billion in 1998 [1]. The incidence of AUGIB has not decreased among the elderly over the last few decades [2]. Because of the baby-boomer phenomena, the health system will manage more elderly patients presenting with AUGIB. This will have significant financial consequences.

We conducted a retrospective study to roughly estimate the cost of hospitalizing elderly patients presenting with AUGIB in the USA. We included all patients 60 years old or older that presented with symptoms of AUGIB to a community hospital from January 2006 to August 2007. AUGIB was defined as hematemesis, coffee ground emesis, or proven bleeding on esophagogastroduodenoscopy. We included 113 patients (62 males and 61 females).

Our study found that the average cost of hospitalizing patients who are 60 years or older presenting with AUGIB is around $\$ 5,485$ per admission. This figure was obtained using the Diagnosis-Related Group (DRG) system.

It is estimated that the annual incidence of AUGIB for patients who are 60 years old or older is 545 cases per 100,000 persons [2]. The total number of persons in the USA who were 60 years old or older in the year 2000 was $45,851,200$ (16.29\%) [3]. In December of 2007, the total
USA population was estimated to be close to 303 million people, hence it is estimated that there were at least $49,358,700$ persons who were 60 years or older in the USA around the same time [4]. Based on the previous figures, we estimate that 269,005 patients older than 59 years will be hospitalized for AUGIB in the USA in 2008. The cost of hospitalizing these patients will be around $\$ 1.48$ billion. This figure is just a rough estimate and there are many factors that may overestimate or underestimate this number. This paper underscores the economic burden associated with AUGIB in the elderly population.

\section{References}

1. Johanson JF. Curbing the costs of GI bleeding. Am J Gastroenterol. 1998;93(8):1384-1385.

2. van Leerdam ME, Vreeburg EM, Rauws EA, et al. Acute upper GI bleeding: did anything change? Time trend analysis of incidence and outcome of acute upper GI bleeding between 1993/1994 and 2000. Am J Gastroenterol. 2003;98(7):1494-1499. doi:10.1111/ j.1572-0241.2003.07517.x.

3. http://www.census.gov/prod/cen2000/dp1/2kh00.pdf. Accessed in October 2007.

4. http://www.census.gov/. Accessed in December 23, 2007.

A. A. Alkhatib - S. M. Abubakr · F. A. Elkhatib Division of Hospitalist, Holy Family Hospital, Washington State University, Spokane, WA, USA 